\title{
Multifaceted Optimization of MSC-Based Formulation upon Sodium Iodoacetate-Induced Osteoarthritis Models by Combining Advantageous HA/PG Hydrogel and Fluorescent Tracer
}

\author{
Ai-tong Wang, ${ }^{1}$ Meng Zhao, ${ }^{1}$ Ying Feng, ${ }^{1}$ Honghong Jia, ${ }^{1}$ Leisheng Zhang $\mathbb{D},{ }^{2,3,4,5}$ Hao Yu $\mathbb{D},{ }^{1}$ \\ Zongjin $\mathrm{Li}^{2}{ }^{2}$ Zhibo Han, ${ }^{1,4}$ and Zhongchao Han ${ }^{1,3,4,5}$ \\ ${ }^{1}$ Cell Products of National Engineering Center \& National Stem Cell Engineering Research Center, Tianjin IMCELL Stem Cell and \\ Gene Technology Co., Ltd., Tianjin, China \\ ${ }^{2}$ The Postdoctoral Research Station, School of Medicine, Nankai University, Tianjin, China \\ ${ }^{3}$ Precision Medicine Division, Health-Biotech (Tianjin) Stem Cell Research Institute Co., Ltd., Tianjin 301700, China \\ ${ }^{4}$ State Key Laboratory of Experimental Hematology \& National Clinical Research Center for Blood Disease, Institute of Hematology \& \\ Blood Diseases Hospital, Chinese Academy of Medical Sciences \& Peking Union Medical College, Tianjin, China \\ ${ }^{5}$ Jiangxi Research Center of Stem Cell Engineering, Jiangxi Health-Biotech Stem Cell Technology Co., Ltd., Shangrao 334000, China
}

Correspondence should be addressed to Leisheng Zhang; zhangleisheng@health-biotech.com and Hao Yu; yuhao@amcellgene.com

Received 12 April 2020; Revised 23 June 2020; Accepted 6 July 2020; Published 23 January 2021

Academic Editor: Alessandro Faroni

Copyright (C) 2021 Ai-tong Wang et al. This is an open access article distributed under the Creative Commons Attribution License, which permits unrestricted use, distribution, and reproduction in any medium, provided the original work is properly cited.

\begin{abstract}
Owing to the boundedness of conventional remedies upon articular cartilage for self-rehabilitation and the incrementally senior citizens, the incidence of osteoarthritis (OA) is increasing worldwide. Empirical studies have revealed the advantageous and promising potentials of mesenchymal stem/stromal cells (MSCs) on the refractory OA, whereas the deficiency of systematic and detailed exploration of MSC-based therapy largely hampers the large-scale applications in regenerative medicine. Herein, we initially utilized the monosodium iodoacetate- (MIA-) induced OA rabbit models and investigated the therapeutic effect of human umbilical cord-derived UC-MSCs at serial dose gradients with the splendid hyaluronic acid and/or propylene glycol hydrogels (HA, HA/PG), respectively. Afterwards, we turned to a dual-luciferase reporter tracing system and evaluated the spatiotemporal distribution and metabolokinetics of bifluorescence expressing UC-MSCs (BF-MSCs) in OA rats. Of the aforementioned trials, we verified that the combination of HA/PG and middle-dose MSCs $\left(0.5 \times 10^{7}\right.$ cells $\left./ \mathrm{ml}\right)$ eventually manifested the optimal efficacy on OA rabbits. Furthermore, with the aid of the bioluminescence imaging (BLI) technology for dynamic in vitro and in vivo tracking, we intuitively delineated the spatiotemporal distribution and therapeutic process of BF-MSCs in OA rats, which substantially confirmed the reinforcement of HA/PG on BF-MSCs for OA treatment. Collectively, our data conformably demonstrated that the middle dose of UC-MSCs combined with HA/PG hydrogel was sufficient for optimal MSC-based formulation for blocking OA progression and promoting cartilage repair, which supplied overwhelming new references and enlightened MSC-based therapeutic strategies for cartilage defects.
\end{abstract}

\section{Introduction}

Osteoarthritis $(\mathrm{OA})$ is acknowledged as the most prevalent and frequent chronic disease of degenerative joints that attributes to multidimensional factors such as aging, overweight, trauma, congenital abnormality, and joint deformity and, in particular, the increase in life expectancy $[1,2]$. Despite the dramatic progress and tremendous effects have been made by multidisciplinary strategies, including surgeries (e.g., microfracture and mosaicplasty), antiinflammatory drugs (e.g., NSAIDs), joint lubricants (e.g., hyaluronic acid), and even the cell-based therapies (e.g., autologous chondrocyte implantation), yet the limitation of self-repair and regeneration capacity in OA individuals still hinders the remission of the progressive degeneration of articular cartilage [3-5]. 
Mesenchymal stem/stromal cells (MSCs) are heterogeneous and immune-privileged populations with multidirectional differentiation potential (e.g., adipocytes, osteoblasts, chondrocytes, and angiocellular), which are therewith recognized as the uppermost components in the hematopoietic microenvironment as well as advantaged sources for translational medicine $[6,7]$. For decades, we and other investigators have illuminated the identification and application of the advantageous and promising sources from adult and perinatal tissues together with human pluripotent stem cells (hPSCs) for the treatment of refractory and recurrent disorders such as aplastic anemia, acute-on-chronic liver failure (ACLF), fistulizing Crohn's disease, acute myocardial infarction (AMI), critical limb ischemia (CLI), and coronavirus disease 2019- (COVID-19) induced acute respiratory distress syndrome (ARDS) [8-13]. State-of-the-art updates have indicated the possibility of MSCs as an alternative option for cartilage repair, especially the allogeneic UC-MSCs with advantageous superiorities in proliferation and immunomodulation and noninvasiveness $[12,14]$. Although administration of OA in preclinical trials by utilizing UC-MSCs has been partially achieved, yet the resumption of the functionally competent articular cartilage is far from satisfaction, let alone the meticulous evaluation of MSCs upon safety and efficacy, which is also the prerequisite for large-scale application in OA treatment $[4,15]$.

For the purpose, we incipiently took advantage of the well-established MIA-induced OA rabbit model and verified the optimally curative effect by comparing the combination schemes among HA/PG and/or UC-MSCs at various gradients (low, middle, or high dose). With the aid of systematic and detailed assessments including clinicopathological observation of articular defects and disease score of multiple staining as well as the comprehensively principal component analysis (PCA), we finally clarified the optimal efficacy by the middle dose of UC-MSC and HA/PG composite. Thereafter, we employed the abovementioned composite for spatiotemporal assessment of the ameliorative effect on the MIAinduced osteoarthritis rat model based on the BLI signal of the dual-luciferase reporter tracing system. Taken together, we introduced multifaceted optimization of formulation for dissecting OA treatment by a moderate dose of UC-MSC and advantageous HA/PG hydrogel composite.

\section{Materials and Methods}

2.1. Rabbits. According to Wang et al. and Hartman et al. reports, with the increase of age and the period of osteoarthritis, the biomechanics and metabolic capacity of articular chondrocytes obviously degenerate. Old rabbits are prone to cartilage degeneration, which affects the consistency and pathological changes of the models [16, 17]. Hence, 28 healthy New Zealand white male rabbits $(2.0 \pm 0.5 \mathrm{~kg})$ were purchased and quarantined for further experimental analyses (YUDA Biotechnology Co., Ltd, Tianjin, China). All procedures were approved by the Ethics Committee of Eye Hospital of Tianjin Medical University (approval number: TJYY2018061114), and the in vivo fluorescence tracer experiments were performed with SD rats and approved by the
Institutional Animal Care and Use Committee of Institute of Radiation Medicine, Chinese Academy of Medical Sciences \& Peking Union Medical College. For each SD rat, anesthesia was conducted with isoflurane; both legs were then injected by using MIA as previously described $[18,19]$. The total volume of UC-MSCs $\left(100 \mu \mathrm{l}\right.$ per joint, $0.5 \times 10^{6}$ cells $/ \mathrm{mL}$ ) was injected into the joint of the knee. Meanwhile, we conformed that all procedures on rabbits and rats were performed in accordance with the Helsinki Declaration of 1975 (as revised in 2008) concerning Human and Animal Rights.

2.2. MIA-Induced Osteoarthritis Rabbit Models. The MIAinduced osteoarthritis rabbit model was displayed as previously reported with several modifications [15, 20, 21]. Briefly, 28 rabbits were randomly divided into seven groups as the following: (i) negative control group (NC, without any treatment; $n=4$ ), (ii) saline group (MIA-induced OA, with saline injection; $n=4$ ), (iii) HA group (MIA-induced OA, with HA injection; $n=4$ ), (iv) HA/PG group (MIA-induced OA, with HA/PG injection; $n=4$ ), (v) HA/PG+UC-MSC (high) group (MIA-induced OA, with $\mathrm{HA} / \mathrm{PG}$ and $1.0 \times 10^{7}$ cells $/ \mathrm{ml}$ injection; $n=4$ ), (vi) $\mathrm{HA} / \mathrm{PG}+\mathrm{UC}-\mathrm{MSC}$ (middle) group (MIA-induced OA, with $\mathrm{HA} / \mathrm{PG}$ and $0.5 \times 10^{7}$ cells $/ \mathrm{ml}$ injection; $n=4$ ), and (vii) $\mathrm{HA} / \mathrm{PG}+\mathrm{UC}-\mathrm{MSC}$ (low) group (MIA-induced OA, with HA/PG and $0.1 \times 10^{7}$ cells $/ \mathrm{ml}$ injection; $n=4)$. All applicable institutional and/or national guidelines for the care and use of animals were followed. The detailed procedures and primer sequences were available in Supplementary Information: Supplementary Procedures and Supplementary Table S1.

2.3. Preparation of UC-MSC and HA/PG Hydrogel Composite. UC-MSCs were purchased from the cell products of the National Engineering Center \& National Stem Cell Engineering Research Center (Tianjin IMCELL Stem Cell and Gene Technology Co., Ltd., Tianjin). Various concentrations of UC-MSCs at passage three $\left(0.1 \times 10^{7}\right.$ cells $/ \mathrm{ml}, 0.5 \times 10^{7}$ cells $/ \mathrm{ml}$, and $1.0 \times 10^{7}$ cells $/ \mathrm{ml}$ ) were thoroughly mixed with $1 \%$ sodium hyaluronate/propylene glycol (2:1, Shandong Freda Biotechnology Co., Ltd, China; HA: $M_{\mathrm{w}}=1579450 \mathrm{Da}$, with the requirements of Chinese Good Manufacturing Practices for Pharmaceutical Products) for MSC/hydrogel composite generation. The detailed procedures and antibodies for FCM assay were available in Supplementary Information: Supplementary Procedures and Supplementary Table S1-S2.

2.4. Macroscopic Analysis. Macroscopic analysis of femoral condyles and tibial plateau in OA rabbits was conducted according to the criteria as reported [22]. In detail, the aforementioned knee joints were carefully collected after euthanasia for the examination of the severe inflammation or extensive fibrosis in the joint. Then, the degree of femur and tibia condyle repair of each rabbit was macroscopically assessed by means of the modified version of the Osteoarthritis Research Society International (OARSI) scoring system [22]. In detail, 3 independent observers graded the OARSI by utilizing the randomized and blind methods. The consistency of these observers was determined by the intragroup correlation coefficient, which was used for 
the assessment of the correlation between the same single pathological section.

2.5. Histopathological Analysis. The preparation for histopathological analysis was displayed as we recently reported with several modifications [6]. Full-thickness samples (including femoral condyles and tibial plateau) were orderly fixed, decalcified, paraffin-embedded, and deparaffinized. For evaluation of $\mathrm{OA}$, microphotographs of the sections were taken using a light microscope (Olympus, BX51, Japan) after hematoxylin and eosin (H\&E), Safranin-O/fast green or Alcian blue staining (Beijing Solarbio Science \& Technology Co., Ltd. China), respectively. The grade of osteoarthritic change was assessed based on the staining areas according to Mankin's score and the formula: SEM percentage $=($ SEM/Mean $) \times 100$ [23]. The detailed procedures and qRT-PCR analysis were available in Supplementary Information: Supplementary Procedures and Supplementary Table S1.

2.6. Cytokine Expression in Synovial Fluid of OA Rabbits. To detect OA-associated inflammatory cytokine and growth factor expression, synovial fluid was collected from each knee joint of rabbits. Firstly, we conducted the preparation of synovial fluid as the following: 20x dilution of washing solution: 1:20 dilution with double distilled water (e.g., $1 \mathrm{ml}$ of concentrated washing solution was added with $19 \mathrm{ml}$ of double-distilled water). Then, the concentrations of interleukin-6 (IL-6), interleukin-1 $\beta$ (IL-1 $\beta$ ), transforming growth factor- $\beta 1$ (TGF- $\beta 1$ ), and matrix metalloproteinases13 (MMP-13) were detected with commercial enzymelinked immunosorbent assay (ELISA) kits (R\&D Systems, Inc., USA) according to the manufacturer's instructions as we described recently $[12,24]$. The detailed information was available in Supplementary Information: Supplementary Table S3.

2.7. Principal Component Analysis (PCA). PCA was used to reveal the internal structure of the present multivariate dataset as a set of coordinates in the corresponding highdimensional data space as we and other investigators reported $[6,25]$. The effects of treatment groups and their correlation with the other characteristics were determined statistically by PCA based on the XLSTAT software (Addinsoft, New York, USA).

2.8. In Vivo Tracking of BF-MSCs (with/without HA/PG) in $O A$ Rats. In vivo tracking of BF-MSCs in SD rats was conducted based on the bioluminescence imaging (BLI) signal $\left(\mathrm{p} / \mathrm{s} / \mathrm{cm}^{2} / \mathrm{sr}\right)$ as we and our collaborators recently reported with several modifications $[11,26]$. BF-MSCs with firefly luciferase (Fluc) and green fluorescent protein (GFP) expression were prepared in D-luciferin $(33 \mu \mathrm{l} / \mathrm{mg}$; Cayman Chemical, USA) before intra-articular injection. Then, a total volume of BF-MSCs (100 $\mu \mathrm{l}$ per joint, $0.5 \times 10^{6}$ cells $\left./ \mathrm{ml}\right)$ was injected into each knee joint. Images were acquired $1 \mathrm{~h}, 48 \mathrm{~h}$, $96 \mathrm{~h}$, and $144 \mathrm{~h}$ with an exposure time of $10 \mathrm{~min}$. The detailed procedures were available in Supplementary Information: Supplementary Procedures. All applicable institutional and/or national guidelines for the care and use of animals were followed.
2.9. Statistical Analysis. All statistical analyses were performed as we described before $[12,27,28]$. The experiments were performed in triplicate for three times, and data were shown as mean \pm standard deviation (SD). For the purpose of dissecting the difference between multiple parameters (including Anderson-Darling test, D'Agostino-Pearson omnibus normality test, Shapiro-Wilk normality test, and KolmogorovSmirnov normality test with Dallal-Wilkinson-Lilliefor $P$ value) of knee joints (8 knee joints in 4 rabbits per group), we primitively checked the Gaussian distribution and found that their respective $P$ value summary was NS, which satisfied the criteria of single-factor analysis of variance. Thus, one-way analysis of variance (ANOVA) was used for statistical analysis. $P$ values were considered significantly when $P<0.05 .{ }^{*} P<0.05$, ${ }^{* *} P<0.01,{ }^{* * *} P<0.001$, and ${ }^{* * * *} P<0.0001$; NS means not significant.

\section{Results}

3.1. Moderate UC-MSC and HA/PG Composite Effectively Ameliorated Pathological Damage of Articular Cartilage in $O A$ Rabbits. For the purpose of systematically and meticulously dissecting the curative effect and optimizing the MSC-based formulation upon osteoarthritis, we primordially took advantage of the well-established sodium iodoacetateinduced rat model $[15,20,21]$ (Figure 1(a)). After euthanasia at week 13, the intact femur and tibia of the knee joints in rabbits among the indicated groups were carefully obtained by surgical operations as described in Materials and Methods. Distinguish from those in the saline group (OA rabbit with saline injection), the joint surface of tibia showed partially ameliorated pathological damage of articular cartilage after injection of UC-MSCs or HA/PG hydrogel, especially OA rabbits with $\mathrm{HA} / \mathrm{PG}+$ high or HA/PG+middle dose UCMSC administration (Figure 1(b)). Similarly, according to the gross morphological observations upon cartilage abrasion and subchondral bone exposure, MIA-induced OA rabbits with intra-articular injection of $\mathrm{HA} / \mathrm{PG}+$ high and $\mathrm{HA} / \mathrm{PG}+$ middle dose UC-MSCs manifested comparable restorative function in the femur as well (Figure 1(c)).

To further quantitatively determinate the ameliorative pathological damage of articular cartilage, we turned to OA scoring based on the OARSI macroscopic evaluation system and confirmed that rabbits with HA/PG+high or HA/PG+middle dose UC-MSC injection showed significantly lower scores than those in the other groups, whereas indistinguishable macroscopic differences were observed between these two groups [22] (Figure 1(d)). Interestingly, we found the OARSI macroscopic score of femurs in rabbits with HA/PG+middle dose UC-MSCs was further decreased compared with the HA/PG+high dose UC-MSC group, which was in consistence with smoother morphological characteristics of joints (Figures 1(d) and 1(e)). Collectively, these data suggested that moderate UC-MSC and HA/PG composite showed superiority in ameliorating macroscopically pathological damage of articular cartilage in OA rabbits.

3.2. Clinicopathological Assessment of the Osteochondral Defects Confirmed the Preferable Efficacy by Moderate UC- 
Establishment of osteoarthritis model and HA/PG/UC-MSC transplantation

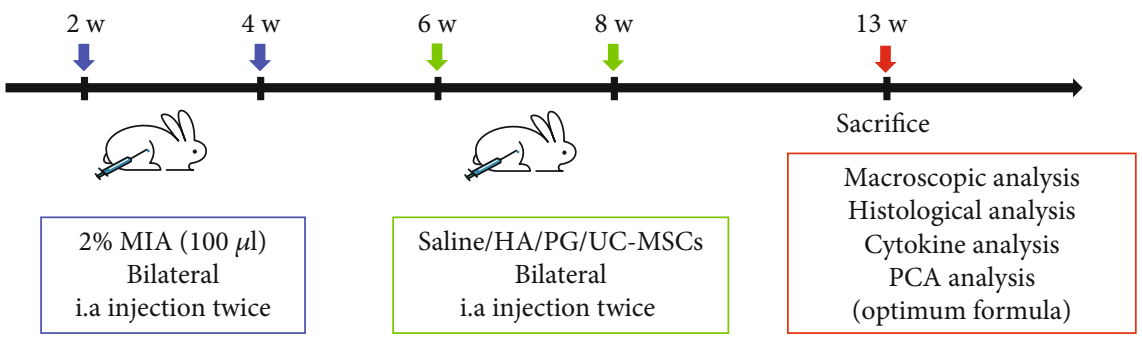

(a)

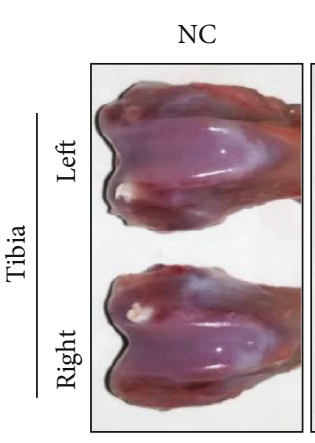

Saline

HA
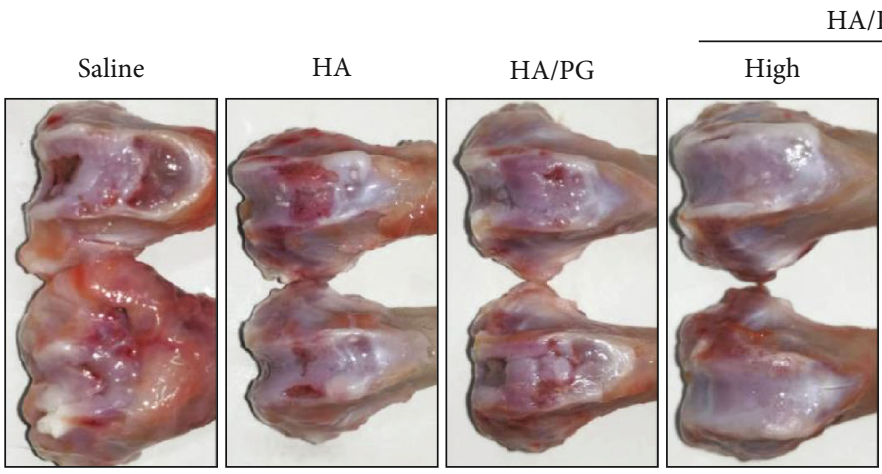

HA/PG+hUC-MSCs (dose)

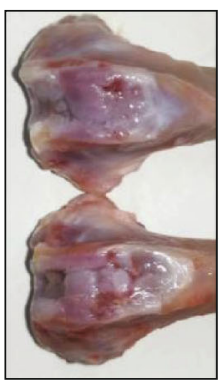

(b)

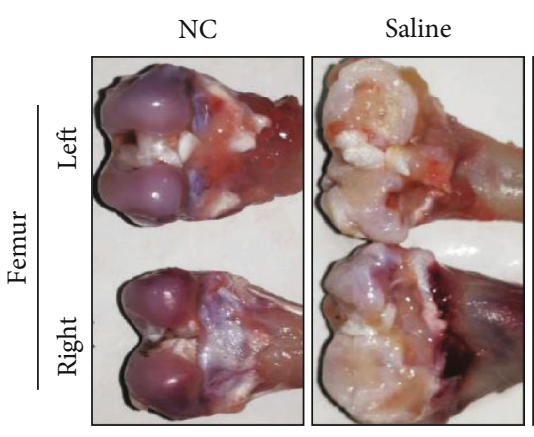

HA

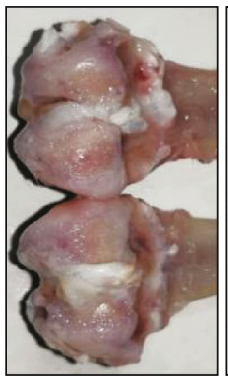

HA/PG

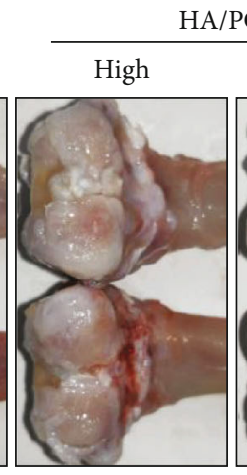

HA/PG+hUC-MSCs (dose)
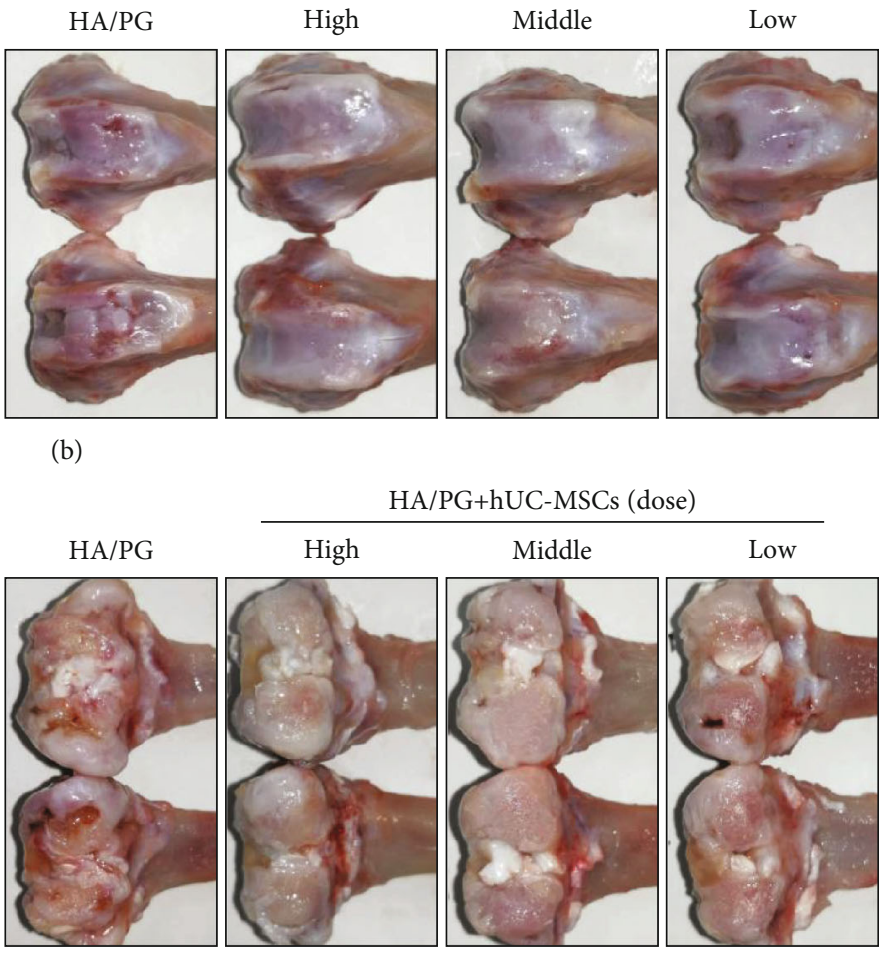

(c)

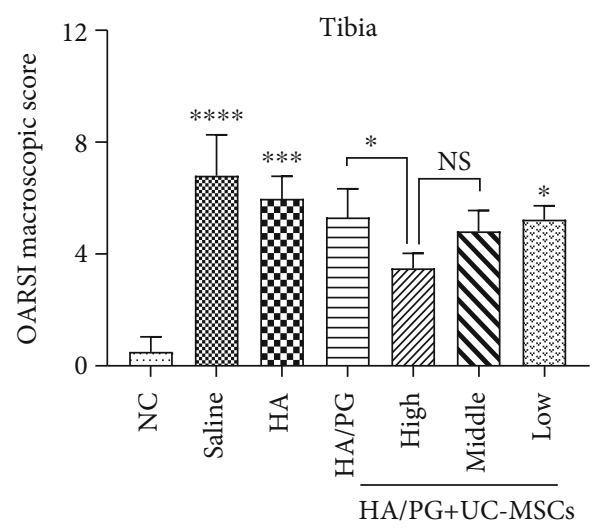

(d)

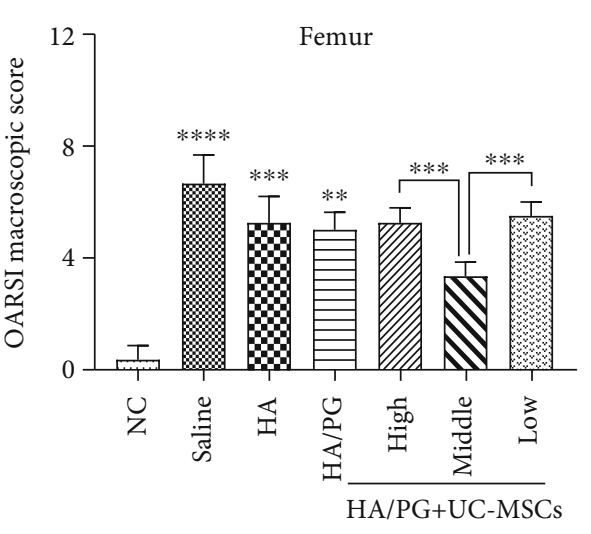

(e)

FIGURE 1: Pathological changes of articular cartilage in OA rabbits with UC-MSC and/or HA/PG composite administration. (a) Schedule of the MIA-induced OA rabbit model with UC-MSC and/or HA/PG composite administration. (b, c) Representative macroscopic images of the tibias (b) and femurs (c) in the indicated groups with intra-articular (i.a.) injection of saline, HA, HA/PG, high/middle/low dose of UC-MSCs+HA/PG hydrogels at week 13, respectively. Rabbits without any treatment were served as negative controls (NC). (d, e) Statistical analysis of the OARSI macroscopic score of tibias (d) and femurs (e) in the aforementioned 7 groups. ${ }^{*} P<0.05$; ${ }^{*} P<0.01$; ${ }^{* * *} P<0.0001 ;{ }^{* * *} P<0.00001 ;$ NS: not significant. 


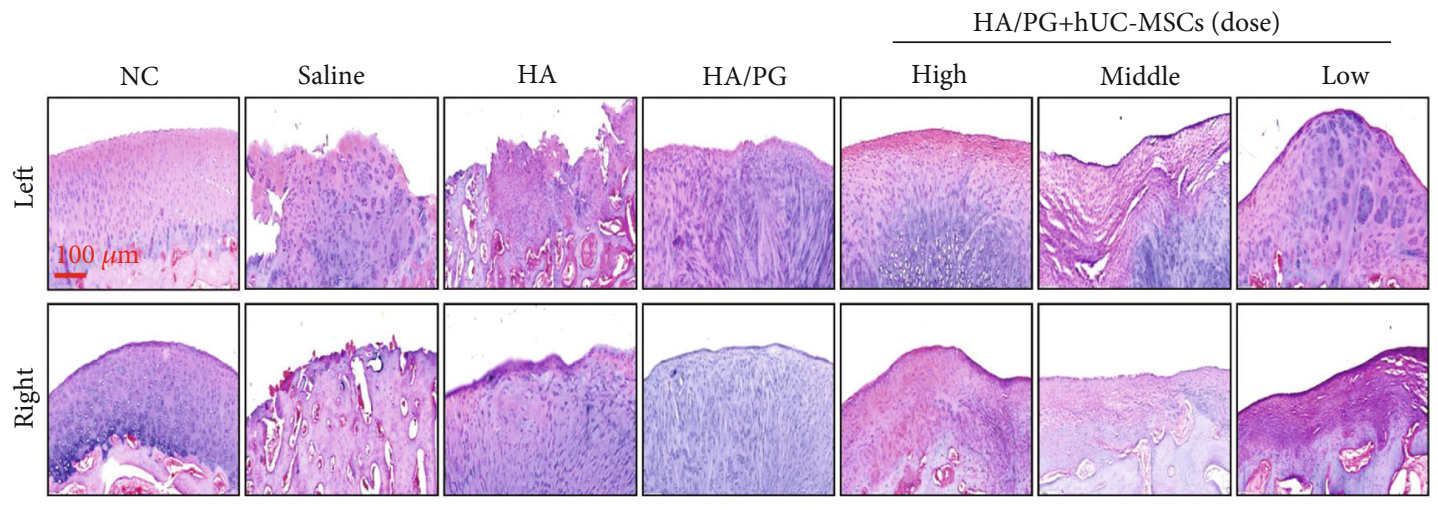

(a)

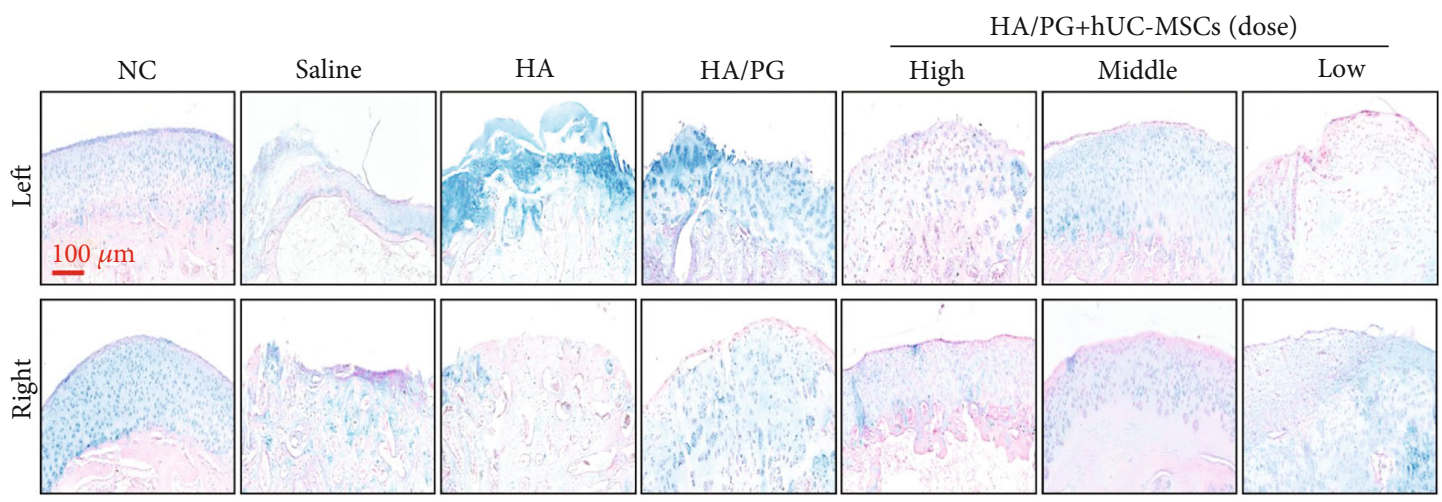

(b)

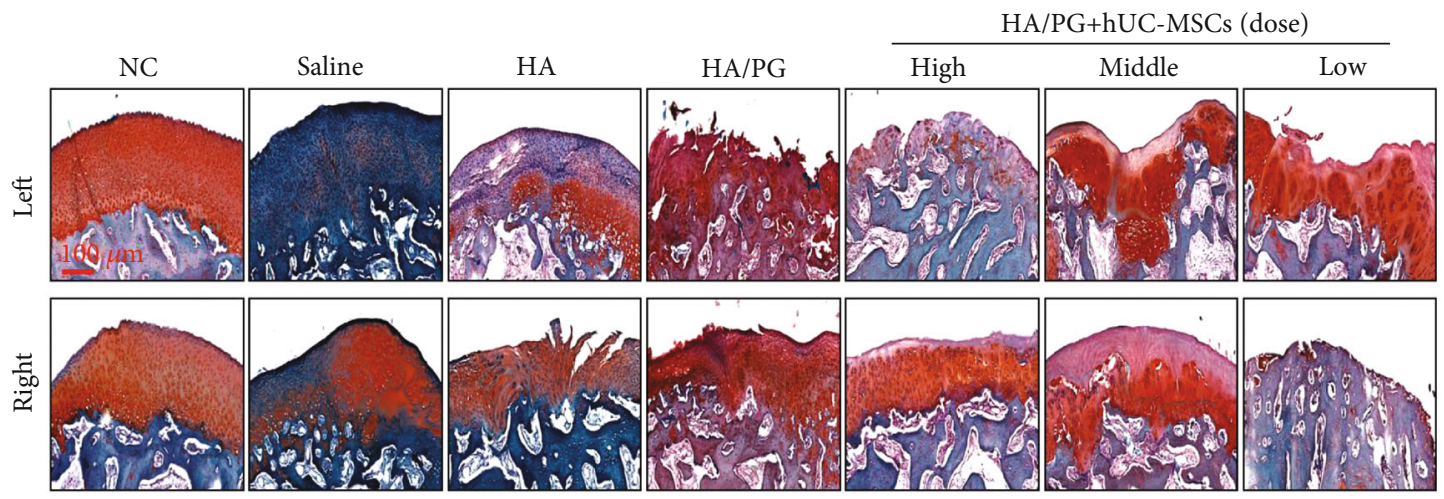

(c)

FIGURE 2: Clinicopathological assessment of the osteochondral defects in rabbits with UC-MSC and/or HA/PG composite administration. $(\mathrm{a}-\mathrm{c})$ Representative histological images of the articular cartilage in the indicated groups with H\&E staining (a), Alcian blue staining (b), and Safranin-O/fast green staining (c), respectively. Scale bar $=100 \mu \mathrm{m}$. The surface changes and loss of collagen and proteoglycans were shown by the microphotographs.

MSC and HA/PG Composite. Having preliminarily identified the ameliorative efficacy of moderate UC-MSC and HA/PG composite in macroscopically pathological damage, we subsequently analyzed the potential distinctions among the indicated groups at a histological level. As shown by the representative photomicrographs of femoral condyles and tibial plateau with $\mathrm{H} \& \mathrm{E}$, Alcian blue, and Safranin-O/fast green staining, rabbits with $\mathrm{HA} / \mathrm{PG}+$ middle or $\mathrm{HA} / \mathrm{PG}+$ high dose UC-MSC treatment exhibited less cartilage loss and surface irregularity of the joints as well as much typical and homogenous articular cartilage structures including smooth, continuous surfaces and strong proteoglycan staining (Figures 2(a)-2(c)). In con- trast, distinguish from the abovementioned two groups and the NC group, the surface irregularity, fibrillation or cleft, changes in cellularity, and loss of tidemark were observed in varying degrees among the other MIA-induced OA groups (saline, HA, or HA/PG group) (Figures 2(a)-2(c)).

Furthermore, quantitative analyses of the histopathologic sections of articular cartilage in OA rabbits were performed by measurement of the percentages of areas with specific staining as we previously reported and also described in the supplementary information [25]. As shown by the calculations based on the aforementioned H\&E staining, HA/PG hydrogel combined with either dose (low, middle, and high) 


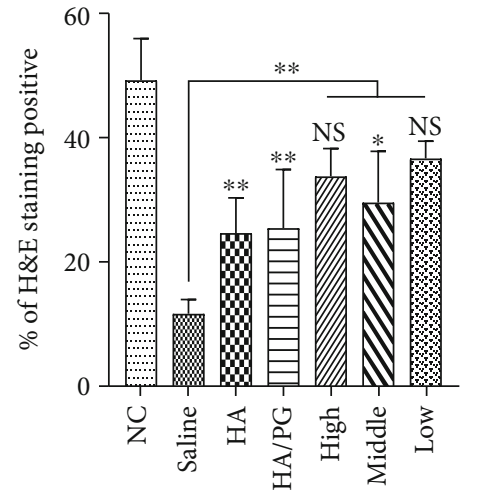

HA/PG+UC-MSCs

(a)

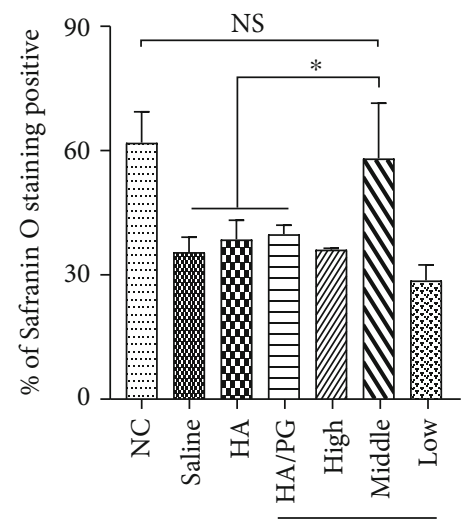

$\mathrm{HA} / \mathrm{PG}+\mathrm{UC}-\mathrm{MSCs}$

(c)

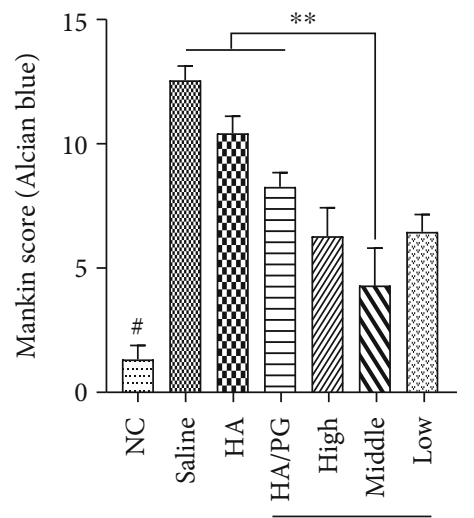

HA/PG+UC-MSCs

(e)

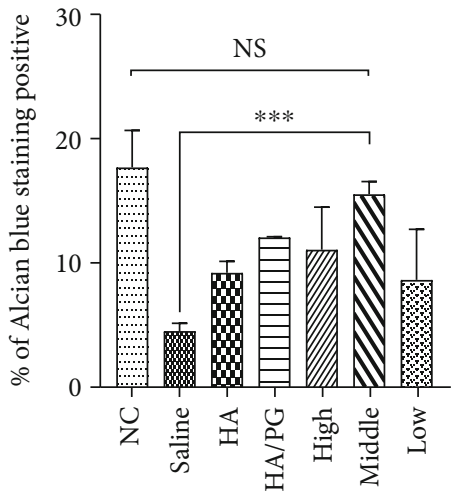

$\mathrm{HA} / \mathrm{PG}+\mathrm{UC}-\mathrm{MSC}$

(b)

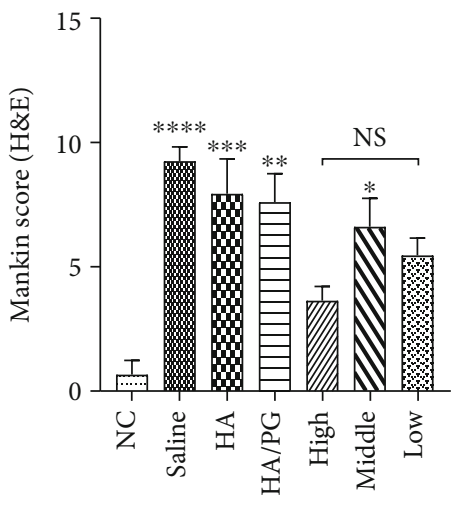

$\mathrm{HA} / \mathrm{PG}+\mathrm{UC}-\mathrm{MSC}$

(d)

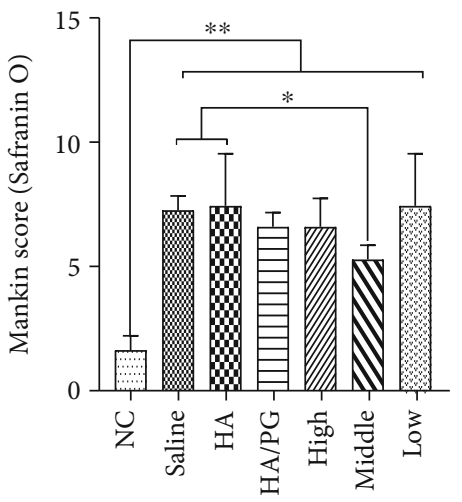

HA/PG+UC-MSCs

(f)

FIgURe 3: Evaluation of the ameliorative effects of OA rabbits by UC-MSC and/or HA/PG composite treatments. (a-c) Quantitative analysis of the percentage of positive stain area based on H\&E staining (a), Alcian blue staining (b), and Safranin-O/fast green staining (c), respectively. All data were shown as mean $\pm \mathrm{SD}(n=8) .{ }^{*} P<0.05 ;{ }^{* *} P<0.01 ;{ }^{* * *} P<0.001$; NS: not significant. (d-f) Quantitative analysis of the modified Mankin scores based on H\&E staining (d), Alcian blue staining (e), and Safranin-O/fast green staining (f), respectively. All data were shown as mean $\pm \operatorname{SD}(n=8) .{ }^{*} P<0.05 ;{ }^{* *} P<0.01 ;{ }^{* * *} P<0.001 ;{ }^{* * * *} P<0.0001$; NS: not significant.

of UC-MSCs exhibited a significantly higher percentage of stained areas compared with the saline group, whereas similar to that in the NC group (Figure 3(a)). As to Alcian blue staining, rabbits received HA/PG+middle dose of UC-MSC treatment exhibited a significantly higher proportion of pos- itive area for collagen staining when compared with the saline group. However, no significant differences were observed between HA/PG+middle dose of UC-MSC and NC groups (Figure 3(b)). Consistently, we found the tendency of the proportions of positive areas with Safranin-O 
staining among the indicated groups was similar to that with the Alcian blue staining. That is, rabbits with $\mathrm{HA} / \mathrm{PG}+$ middle dose of UC-MSC administration exhibited a significantly higher percentage of positive area for proteoglycan-associated GAG staining than the other OA groups (Figure 3(c)). Simultaneously, we aimed to further dissect the potentially microscopic alterations of articular cartilage based on the modified Mankin score as well. In regard to the Mankin score of H\&E staining, rabbits with HA/PG hydrogel+UC-MSC injection revealed lower scores than the other groups (saline, HA, or HA/PG) to some extent (Figure 3(d)). Different from those with a high or low dose of UC-MSC treatment, rabbits with middle dose showed further decreases in Mankin scores of Alcian blue and Safranin-O staining, respectively (Figures 3(e) and 3(f)). Taken together, these data further confirmed the preferable efficacy of moderate UC-MSC and HA/PG composite in suppressing clinicopathological changes in OA rabbits.

3.3. UC-MSC and HA/PG Composite Congruously Reinforced the Reduction of Proinflammatory Cytokines in Joint Fluid. Extensive studies have indicated the involvement of multiple proinflammatory cytokines during osteoarthritis for accelerating damage of articular cartilage. Thus, we were curious about whether the ameliorative symptoms of OA were due to the reduction of pivotal proinflammatory cytokines. For the purpose, we detected the indicated groups by utilizing ELISA. Compared with the NC group, we intuitively observed the abnormal elevation of IL-1 $\beta$, IL-6, MMP-13, and TGF- $1 \beta$ expression in the joint fluids in the saline group (MIA-induced OA with saline injection), while the dysupregulation was suppressed by HA/PG and/or UC-MSC treatments and, in particular, further alleviated by the $\mathrm{HA} / \mathrm{PG}+\mathrm{UC}-\mathrm{MSC}$ administration (Figures 4(a)-4(d)). Meanwhile, we also noticed the reinforced inhibitory effects on IL- $1 \beta$ and MMP-13 in the joint fluids by moderate UCMSC and HA/PG composite as well (Figures 4(a)-4(d)).

To comprehensively evaluate the ameliorative effect and explore the optimal formulation upon MIA-induced osteoarthritis, we took advantage of the principal component analysis (PCA) as we and others reported $[6,25]$. Based on the 19 indicated parameters involved in pathological damage of articular cartilage, clinicopathological variations of the osteochondral defects, and proinflammatory cytokines, the Euclidean geometric map revealed the internal structure of the present multivariate dataset as a set of coordinates in the corresponding high-dimensional data space. From the view of PC1 (F1, 46.69\%), rabbits with $\mathrm{HA} / \mathrm{PG}+\mathrm{UC}-\mathrm{MSC}$ (middle) injection displayed closer relationships to the parameters including percentages and Mankin scores of $\mathrm{H} \& \mathrm{E} /$ Safranin-O/Alcian blue staining and TGF- $\beta 1$ compared with other groups (saline, HA, HA/PG, and HA/PG+UCMSCs (low dose)). As to PC2 (F2, 14.51\%), HA/PG+UCMSC (middle) administration was distantly related to the parameters including inflammatory cytokines (IL-6, IL- $1 \beta$, and MMP-13), which indicated the superiority in antiinflammatory property (Figure 4(e)). Collectively, these data further manifested moderate UC-MSC and HA/PG composite held advantageous prospect as an optimal formulation for OA treatment.
3.4. Establishment of Bifluorescence Expressing BF-MSCs with Signatures of Immunophenotype and Multilineage Differentiation. For spatiotemporal dissection of the metabolokinetics of UC-MSCs on OA treatment, we utilized the bioluminescence imaging (BLI) technology with the lentivirus-mediated dual-fluorescence reporter construct (pLV-Fluc-eGFP) as we and collaborators reported recently [11, 26, 29, 30] (Figure 5(a)). After continuous enrichments, UC-MSCs expressing a high level of luci and GFP were established (denoted as BF-MSCs), which also exhibited representative immunophenotype of adult MSCs (Figures 5(b) and $5(c)$ ). Concurrently, by conducting multilineage differentiation detection, we found the BF-MSCs were sufficient to, respectively, generate adipocytes, osteoblasts, and chondrocytes under the indicated conditions as shown by the O Red $\mathrm{O}$, Alizarin Red, and Alcian blue staining and qRT-PCR analysis of adipogenic- (PPAR $\gamma$ and ADIPOQ), osteogenic(RUNX2 and BGLAP), and chondrogenic- (ACAN and SOX9) associated genes (Figures 5(d) and 5(e)). In addition, with the aid of amplification curve and karyotypic analysis, we verified that the BF-MSCs after gene editing exhibited admirable in vitro proliferation and chromosomal stability as well (Figures 5(f) and 5(g)). Taken together, the established BF-MSCs with bifluorescence expression possessed decent signatures for further in vivo analysis.

3.5. BF-MSCs Exhibited Stable Fluorescence Intensity Both In Vitro and In Vivo. To further evaluate the sensitivity of the GFP and Fluc fluorescences in BF-MSCs as well as the potentiation effect of the HA/PG hydrogel, we incipiently performed an in vitro dosage-dependent experiment by seeding cells into 12 -well plates at various density gradients. In consistence with our previous reports, both the GFP and BLI signal intensity of Fluc showed pertinently matched intensity with the density of BF-MSCs in vitro under pretreatment with firefly luciferase substrate D-luciferin (Figures 6(a)-6(c)) [8, 29].

For the purpose of verifying the spatiotemporal metabolokinetics of BF-MSCs as well as the enhanced therapeutic effect of the moderate UC-MSC and HA/PG composite in vivo, we turned to another well-established MIA-induced OA model on SD rats (Figure 6(d)). Different from those in the control group with only UC-MSC injection (denoted as -HA/PG), rats with moderate UC-MSC and HA/PG composite administration showed delayed decline in BLI signal according to the intensity gradients of BLI signal by in vivo imaging systems (IVIS), which suggested the credibility of potentiation effect of $\mathrm{HA} / \mathrm{PG}$ to BF-MSCs. Additionally, the transplanted UC-MSC and HA/PG composite could maintain in the sites of articular cartilage in OA rats and survive for one week (Figures 6(d) and 6(e)). Therefore, the BF-MSCs was also sensitive enough for spatiotemporal distribution and metabolism analysis of cell tracking in vivo, and the HA/PG hydrogel manifested enhanced residence of UC-MSCs upon OA.

\section{Discussion}

Osteoarthritis is a principal and challenging disorder of articular cartilage due to multiple pathogenic factors and a broad 


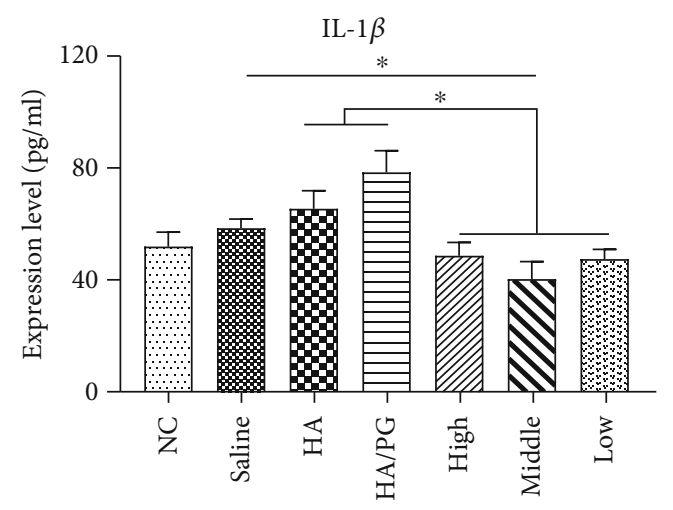

HA/PG+UC-MSCs

(a)

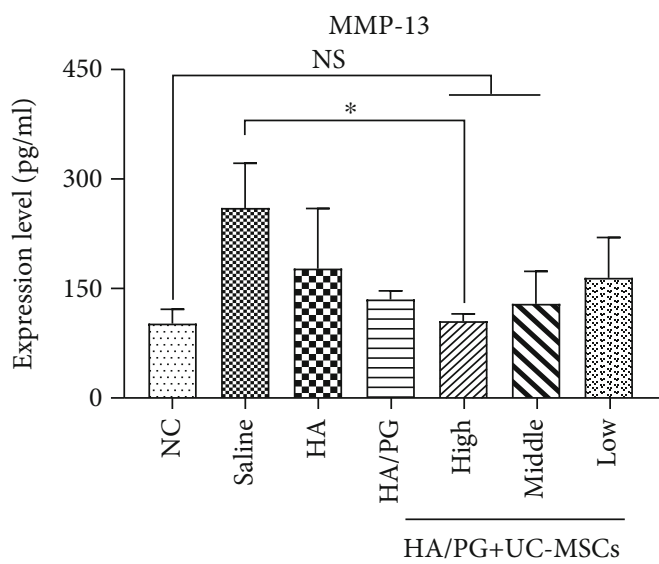

(c)

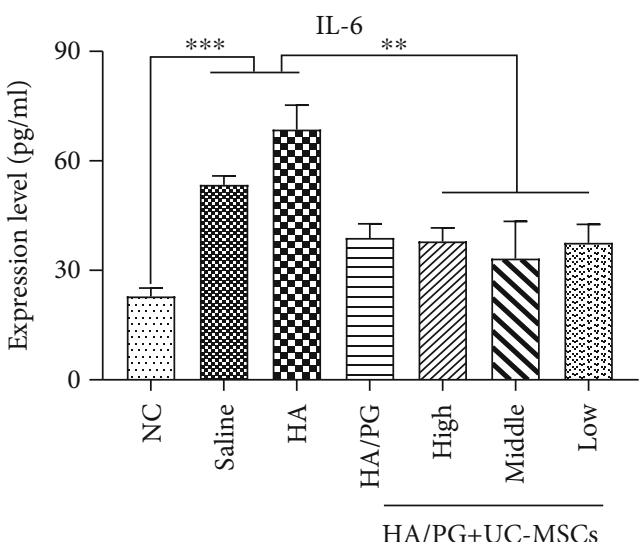

(b)

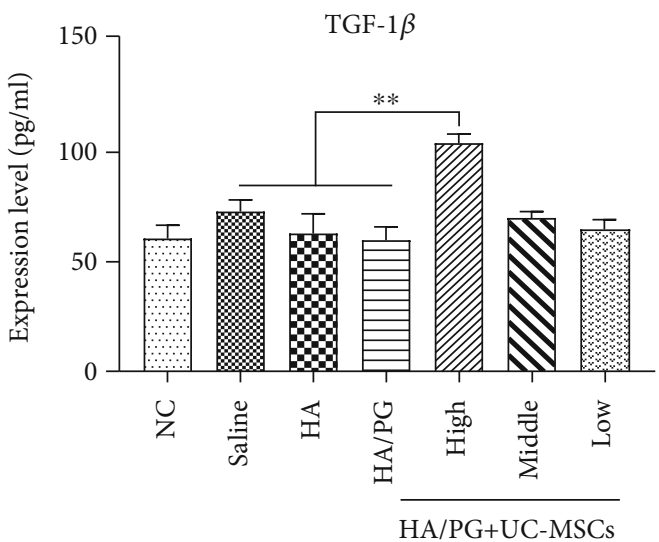

(d)

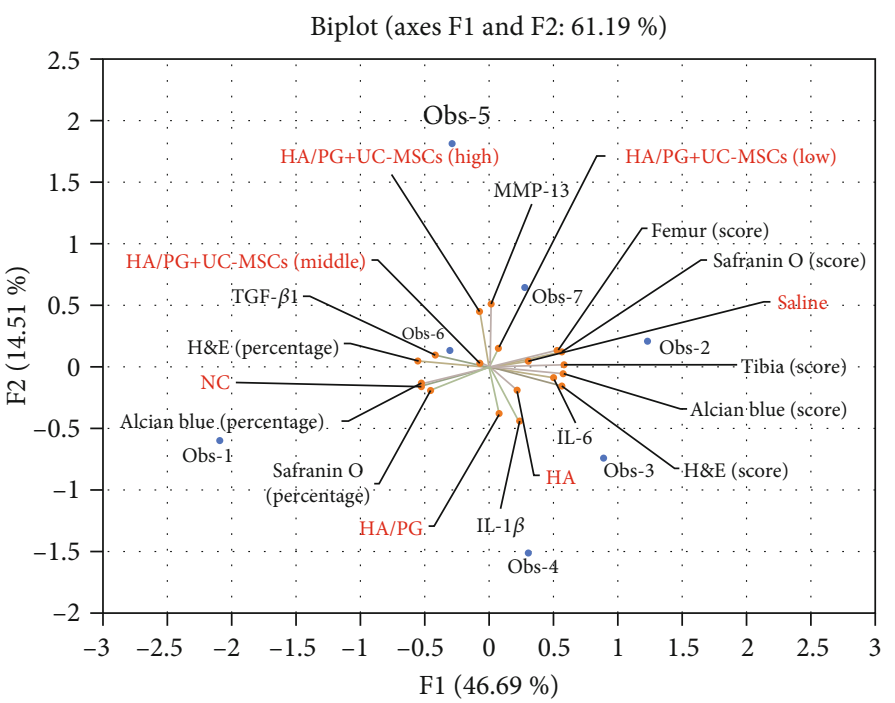

(e)

FIGURE 4: Expressions of proinflammatory cytokines in joint fluid and systematic assessment of OA rabbits. (a-d) Statistical analysis of the expression levels of proinflammatory factors in synovial fluid in the indicated 7 groups by ELISA including IL-1 $\beta$ (a), IL-6 (b), MMP-13 (c), and TGF- $1 \beta(\mathrm{d})$. Data were shown as mean \pm SD $(n=8) .{ }^{*} P<0.05 ;{ }^{* *} P<0.01$; ${ }^{* * *} P<0.001$; NS: not significant. (e) Principal component analysis (PCA) of the aforementioned 19 parameters involved in the MIA-induced OA rabbit model among the indicated 7 groups. F1 and F2 represented principal components 1 and 2 (PC-1 and PC-2), respectively. 


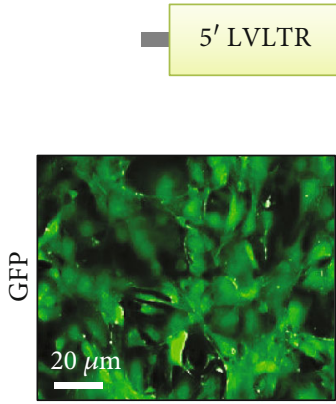

Ubiquitin promoter
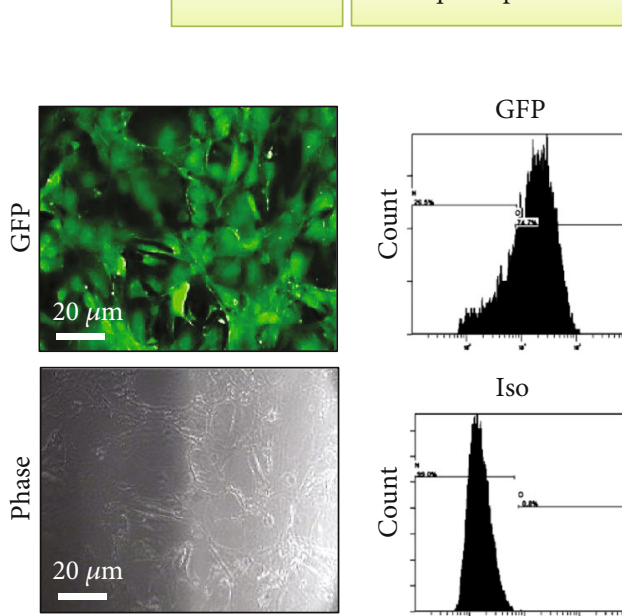

(b)
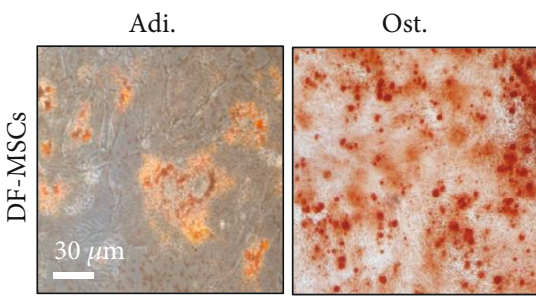

(d)
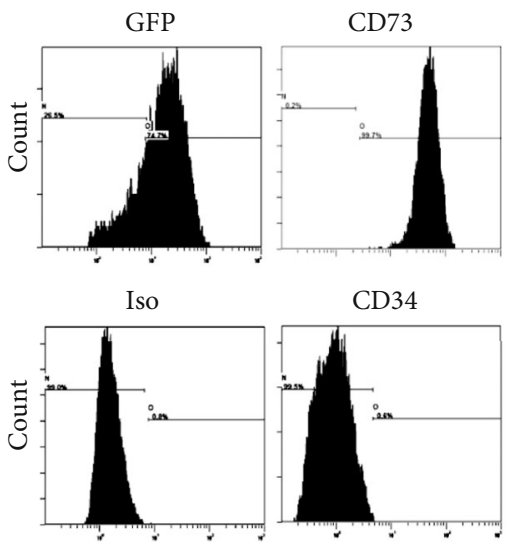

CD73
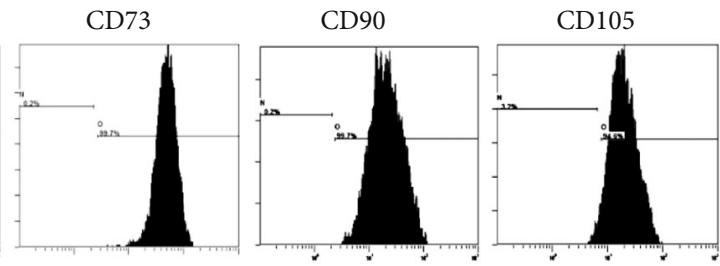

CD45
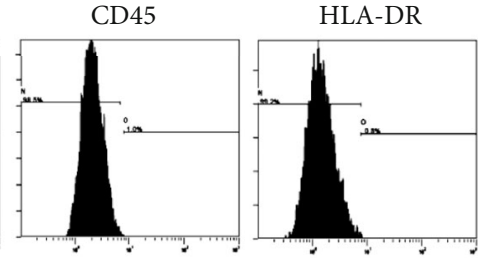

(c)
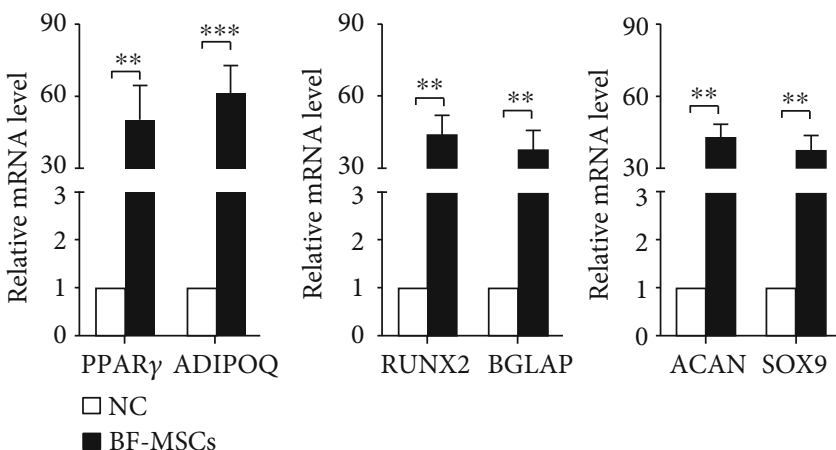

(e)

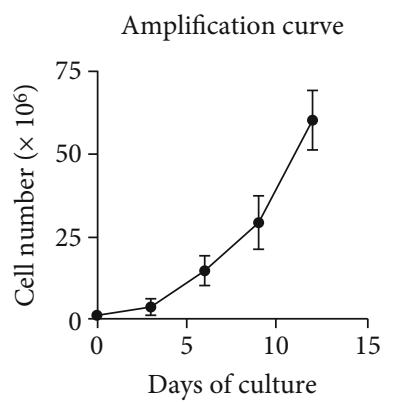

(f)

(g)

FIGURE 5: The multifaceted characterizations of the dual-fluorescence expressing BF-MSCs. (a) Illustration of the lentivirus-mediated dual-fluorescence reporter construct (pLV-Fluc-eGFP). Fluc and GFP were for BLI and green fluorescent protein imaging, respectively. (b) The phase contrast and GFP fluorescence of dual-fluorescence expressing UC-MSCs (denoted as BF-MSCs). (c) FCM analysis of GFP and MSC-associated biomarker in BF-MSCs. (d) Multilineage differentiation potential (adipogenic, osteogenic, and chondrogenic differentiation) of BF-MSC-derived cells (upper panel) and untreated DF-MSCs (bottom panel) identified by Oil Red O (left panel), Alizarin red (middle panel), and Alcian blue (right panel) staining, respectively. Scale bar $=30 \mu \mathrm{m}$. (e) qRT-PCR analysis of the adipogenic- (ADIPOQ and PPAR $\gamma$ ), osteogenic- (RUNX2 and BGLAP), and chondrogenic- (ACAN and SOX9) associated genes in the indicated BF-MSCs (mean $\pm \mathrm{SD}, n=3$ ). ${ }^{* *} P<0.01 ;{ }^{* * *} P<0.001$. (f) Amplification curve of BF-MSCs based on the cumulative cell numbers and days of cultivation (mean $\pm \mathrm{SD}, n=3$ ). (g) Karyotypic analysis of UC-MSCs and BF-MSCs cultured in $10 \%$ FBS/DF12 medium with G-banded chromosome detection.

demographic population, and in particular, the elders are confronting and struggling with the burden of the refractory chronic disease $[2,4,5]$. Even though multifaceted improvements have been achieved by multidisciplinary therapies such as surgical procedures and anti-inflammatory drugs, yet the pathogenesis and efficacy of OA with degeneration and functional losses are still elusively complicated for ascertainment $[3,31]$. Herein, we took advantage of the promising MSC-based cytotherapy and advantageous HA/PG hydrogel composites for optimizing the formulation upon MIA- 
Fluorescent images of BF-MSCs in a dosage-dependent manner (GFP)

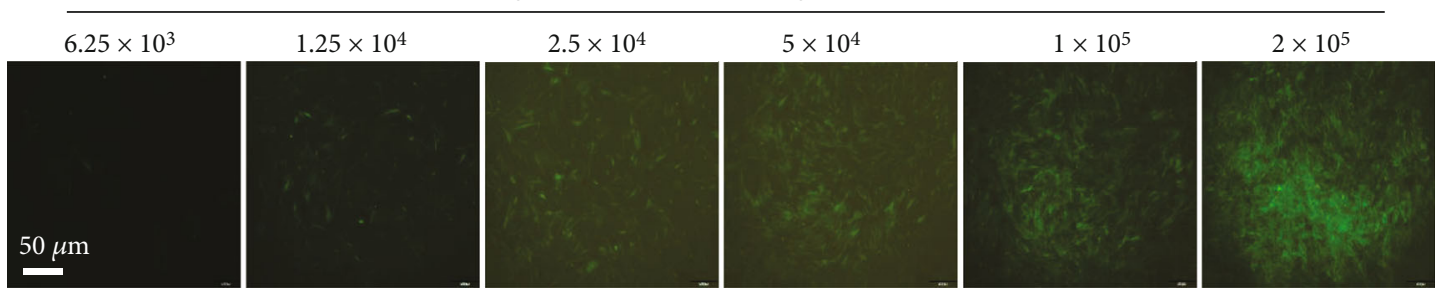

(a)

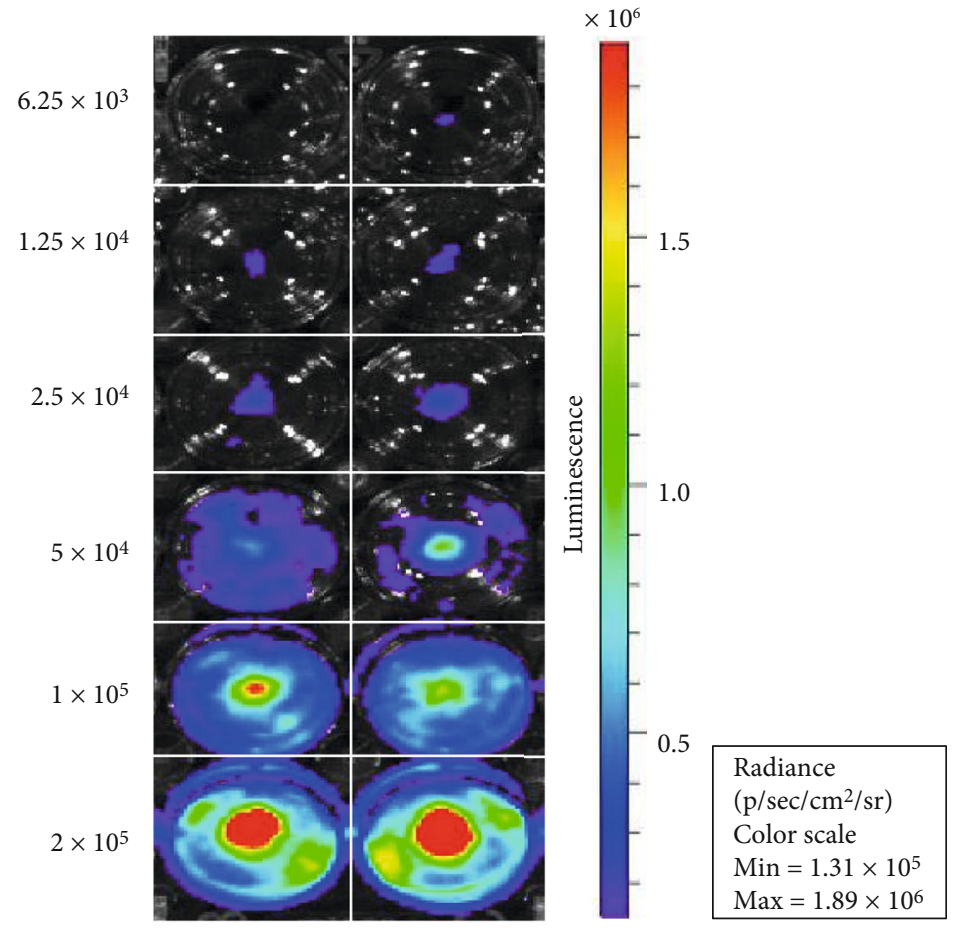

(b)

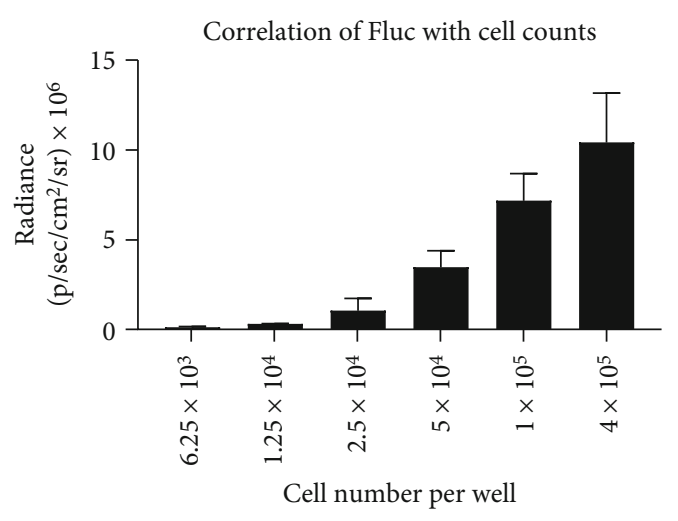

(c)

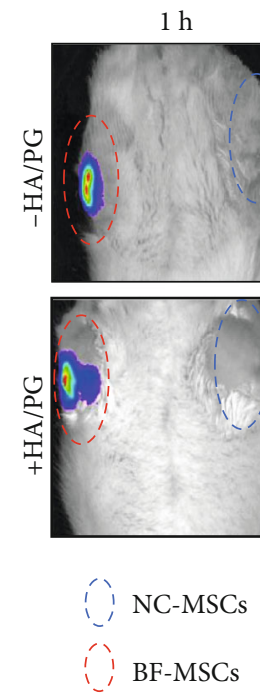

$24 \mathrm{~h}$

$96 \mathrm{~h}$

$144 \mathrm{~h}$

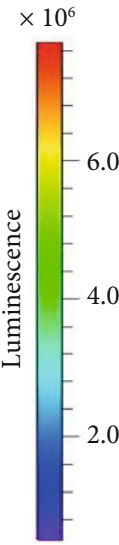

Radiance

$\left(\mathrm{p} / \mathrm{sec} / \mathrm{cm}^{2} / \mathrm{sr}\right)$

Color scale

Min $=5.08 \times 10^{5}$

$\operatorname{Max}=7.72 \times 10^{6}$

(d)

Figure 6: Continued. 


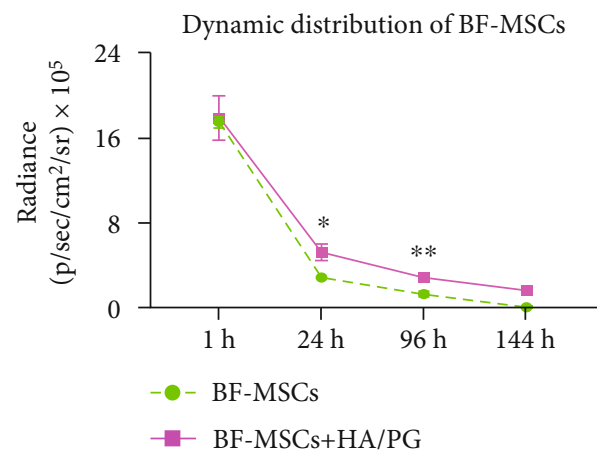

(e)

Figure 6: The dynamic metabolokinetics of BF-MSC or BF-MSC/HA/PG composite in vivo. (a, b) Representative fluorescent images of seeded BF-MSCs with GFP (a) and luci (b) expression at a series of cell density gradients in vitro. (c) The correlation curve based on the BLI signal intensity of Fluc (radiance) and seeded cell counts. (d) Living images of BF-MSCs with or without HA/PG hydrogel composites based on the BLI signal intensity of Fluc in living imager (IVIS system) at the indicated time points. (e) The dynamic distribution curve of BF-MSCs based on cell density gradients and the BLI signal intensity of Fluc in the articular cartilage of OA rats (mean \pm SD, $n=3$ ). ${ }^{*} P<0.05 ;{ }^{*} P<0.01$.

induced osteoarthritis models upon rabbits and rats. Furthermore, aided with the BLI signal, we intuitively and dynamically figured out the spatiotemporal metabolokinetics of MSCs in vivo, which would congruously benefit the fundamental and clinical researches upon the safety and effectiveness assessment of MSC-based cytotherapy.

Of the reported MSCs, UC-MSC is regarded as a preferable source with superiorities including long-term proliferation and immunoregulatory attributes for numerous recurrent and refractory disease remodeling including cartilage regeneration $[12,32,33]$. Meanwhile, we and other investigators recently also reported the discrepant and controversial efficacy of UC-MSCs on disorders such as GVHD, acute liver failure, and MIA-induced osteoarthritis attribute to cell vitality and dosage, respectively [34-38]. Considering the contingent risks in safety and effectiveness, it is of importance and indispensability for systematically and meticulously dissecting the optimal formulation for MSC-based therapy before large-scale clinical applications. Herein, by conducting intra-articular injection of UC-MSCs at various concentration gradients, we found moderate MSC application manifested consistent efficacy but preferable immunoregulatory capacity in suppressing proinflammatory factors in joint fluid. Additionally, the BLI signal of dual-fluorescence expressing BF-MSCs was sufficient for in vivo real-time assessment of cell metabolokinetics.

State-of-the-art progress has highlighted the feasibility and cooperativity of biomaterials with MSCs in regenerative medicine $[39,40]$. For instance, Park et al. reported UCBMSCs and HA hydrogel in repairing articular cartilage lesion in surgery-induced OA rabbit and rat models, respectively $[37,40]$. However, by utilizing a more realistic and intractable MIA-induced model with characteristics including proteoglycan matrix loss and disruption of glycolysis as well as functional joint impairment, van Buul et al. found MSC administration could only partially reduce pain without regaining the beneficial effect on degenerative changes [38].
To overcome the shortcomings, we repeated the MIAinduced OA model both on rabbits and on rats and conducted multifaced optimization of MSC-based formulation with the much more advantageous HA/PG hydrogel, which exhibited enhanced therapeutic efficacy than $\mathrm{HA}$ and approved as a safe and food-grade ingredient by the Food and Drug Administration (FDA) in ameliorating osteoarthritis progression $[41,42]$. Hence, we have conducted a novel composite by moderate UC-MSCs and HA/PG hydrogel to dissect the therapeutic potential in MIA-induced OA models.

Effective evaluations upon safety, effectiveness, and reproducibility in preclinical and clinical studies are the prerequisites for large-scale application of MSC-based therapy, especially the pharmacokinetic and pharmacodynamic characterization in vivo $[14,34]$. However, most of the current methodologies for cell labeling including radioprobes and fluorophores were insufficient for long-term cell monitoring due to the excessive dilution of agents during cellular proliferation and persistence of signals from released agents and dead cells [43, 44]. Conversely, we and other investigators have recently developed an indirect and dual-fluorescent labeling system for in vivo MSC tracking based on the lentivirus-mediated GFP and luciferase (Luc), which is competent for tracer upon exosome vesicle as well [11, 26, 45]. In this study, our data showed that the system was also capable of tracing the spatiotemporal metabolokinetics of UC-MSC+HA/PG composite. Above all, MSCs in HA/PG displayed preferable homing and delayed attenuation in the damaged articular cartilage compared with single $\mathrm{HA} / \mathrm{PG}$ or UC-MSC administration attribute to the splendid viscoelasticity, minimal influence to cell behavior, and the encapsulated microenvironment by HA/PG $[8,11,41,46]$.

\section{Conclusions}

Overall, our data suggested that moderate UC-MSCs and HA/PG composite supplied an optimal formulation upon 
MIA-induced osteoarthritis models and the potential in regenerative medicine for alleviating articular cartilage defects. Meanwhile, the BLI-based system was sufficient for monitoring the dynamic metabolism of UC-MSCs (with/without $\mathrm{HA} / \mathrm{PG}$ ) in vivo, which was promising for the assessment of MSC-based cytotherapy before large-scale application.

\section{Data Availability}

The data used to support the findings of the study are included in the article. Additional data related to this study are also available from the corresponding author.

\section{Conflicts of Interest}

The indicated commercial companies including Health-Biotech (https://www.health-biotech.com/en/intro.html), Amcellgene (https://www.amcellgene.com/en/), and Chase Sun (http:// www.chasesun.cn/) gave technical support, which we have expressed our thanks in the "Acknowledgments" section.

\section{Authors' Contributions}

Ai-tong Wang and Meng Zhao performed the experiments, collection, and assembly of data; Ying Feng, Honghong Jia, Zongjin Li, Zhibo Han, and Zhongchao Han helped with collection and assembly of data; Leisheng Zhang and Hao Yu contributed to conception and design, data analysis and interpretation, manuscript writing, and final approval of the manuscript. All authors read and approved the final manuscript. Ai-tong Wang and Meng Zhao contributed equally to this work.

\section{Acknowledgments}

This work was supported by grants from the Tianjin Science and Technology Project for Overseas Student (JH-20180070802), project funded by the China Postdoctoral Science Foundation (2019M661033), the Natural Science Foundation of Tianjin (19JCQNJC12500), the National Natural Science Foundation of China (81330015), emergency project funded by the Department of Science and Technology of Jiangxi Province (2020, to ZCH), key project funded by the Department of Science and Technology of Shangrao City 2020, to ZCH), and the Natural Science Foundation of Hebei Province (H2020206403). The authors would like to thank Professor Dengke Liu in the enterprise postdoctoral working station of Tianjin Chase Sun Pharmaceutical Co., Ltd. for their suggestions. Also, we thank the postdoctoral workstation of Wuqing Development Zone for their technical support.

\section{Supplementary Materials}

Supplementary Table 1: the primer sequences of indicated genes for quantitative RT-PCR analysis. Supplementary Table 2: the antibodies for flow cytometry analysis. Supplementary Table 3: the ELISA kits used in this study. Supplementary Procedures: the additional procedures accompanied with the main manuscript. Supplementary References: the additional references accompanied with the main manuscript. (Supplementary Materials)

\section{References}

[1] R. Barnett, "Osteoarthritis," Lancet, vol. 391, no. 10134, p. 1985, 2018.

[2] J. W. Bijlsma, F. Berenbaum, and F. P. Lafeber, "Osteoarthritis: an update with relevance for clinical practice," Lancet, vol. 377, no. 9783, pp. 2115-2126, 2011.

[3] A. E. Nelson, "Osteoarthritis year in review 2017: clinical," Osteoarthritis and Cartilage, vol. 26, no. 3, pp. 319-325, 2018.

[4] C. T. Appleton, "Osteoarthritis year in review 2017: biology," Osteoarthritis and Cartilage, vol. 26, no. 3, pp. 296-303, 2018.

[5] D. Pereira, E. Ramos, and J. Branco, "Osteoarthritis," Acta Médica Portuguesa, vol. 28, no. 1, pp. 99-106, 2015.

[6] L. Zhang, H. Wang, C. Liu et al., "MSX2 initiates and accelerates mesenchymal stem/stromal cell specification of hPSCs by regulating TWIST1 and PRAME," Stem Cell Reports, vol. 11, no. 2, pp. 497-513, 2018.

[7] X. Zhang, Y. Yang, L. Zhang et al., "Mesenchymal stromal cells as vehicles of tetravalent bispecific Tandab (CD3/CD19) for the treatment of B cell lymphoma combined with IDO pathway inhibitor D-1-methyl-tryptophan," Journal of Hematology \& Oncology, vol. 10, no. 1, article 56, 2017.

[8] K. Zhang, X. Zhao, X. Chen et al., "Enhanced therapeutic effects of mesenchymal stem cell-derived exosomes with an injectable hydrogel for hindlimb ischemia treatment," ACS Applied Materials \& Interfaces, vol. 10, no. 36, pp. 3008130091, 2018.

[9] M. Shi, Z. Zhang, R. Xu et al., "Human mesenchymal stem cell transfusion is safe and improves liver function in acute-onchronic liver failure patients," Stem Cells Translational Medicine, vol. 1, no. 10, pp. 725-731, 2012.

[10] M. Lim, W. Wang, L. Liang et al., "Intravenous injection of allogeneic umbilical cord-derived multipotent mesenchymal stromal cells reduces the infarct area and ameliorates cardiac function in a porcine model of acute myocardial infarction," Stem Cell Research \& Therapy, vol. 9, no. 1, article 129, 2018.

[11] Y. Wei, H. Hou, L. Zhang et al., "JNKi- and DAC-programmed mesenchymal stem/stromal cells from hESCs facilitate hematopoiesis and alleviate hind limb ischemia," Stem Cell Research \& Therapy, vol. 10, no. 1, article 186, 2019.

[12] J. Huo, L. Zhang, X. Ren et al., "Multifaceted characterization of the signatures and efficacy of mesenchymal stem/stromal cells in acquired aplastic anemia," Stem Cell Research \& Therapy, vol. 11, no. 1, article 59, 2020.

[13] X. He, S. Ai, W. Guo et al., "Umbilical cord-derived mesenchymal stem (stromal) cells for treatment of severe sepsis: aphase 1 clinical trial," Translational Research, vol. 199, pp. 52-61, 2018.

[14] Q. Zhao, L. Zhang, Y. Wei et al., "Systematic comparison of hUC-MSCs at various passages reveals the variations of signatures and therapeutic effect on acute graft-versus-host disease," Stem Cell Research \& Therapy, vol. 10, no. 1, p. 354, 2019.

[15] E. R. Chiang, H. L. Ma, J. P. Wang, C. L. Liu, T. H. Chen, and S. C. Hung, "Allogeneic mesenchymal stem cells in combination with hyaluronic acid for the treatment of osteoarthritis in rabbits," PLoS One, vol. 11, no. 2, article e0149835, 2016.

[16] Z. Wang, J. H. Kim, K. Higashino et al., "Cartilage intermediate layer protein (CILP) regulation in intervertebral discs. The effect of age, degeneration, and bone morphogenetic protein2," Spine, vol. 1976, no. 37, pp. E203-E208, 2012. 
[17] R. Hartman, P. Patil, R. Tisherman et al., "Age-dependent changes in intervertebral disc cell mitochondria and bioenergetics," European Cells \& Materials, vol. 36, pp. 171-183, 2018.

[18] N. Araya, K. Miyatake, K. Tsuji et al., "Intra-articular injection of pure platelet-rich plasma is the most effective treatment for joint pain by modulating synovial inflammation and calcitonin gene-related peptide expression in a rat arthritis model," The American Journal of Sports Medicine, vol. 48, no. 8, pp. 2004-2012, 2020.

[19] H. J. Shin, H. Park, N. Shin et al., "Pink1-mediated chondrocytic mitophagy contributes to cartilage degeneration in osteoarthritis," Journal of Clinical Medicine, vol. 8, no. 11, p. 1849, 2019.

[20] J. E. Kim, D. H. Song, S. H. Kim, Y. Jung, and S. J. Kim, “Development and characterization of various osteoarthritis models for tissue engineering," PLoS One, vol. 13, no. 3, article e0194288, 2018.

[21] L. Grossin, S. Etienne, N. Gaborit et al., "Induction of heat shock protein 70 (Hsp70) by proteasome inhibitor MG 132 protects articular chondrocytes from cellular death in vitro and in vivo," Biorheology, vol. 41, no. 3-4, pp. 521-534, 2004.

[22] S. Laverty, C. A. Girard, J. M. Williams, E. B. Hunziker, and K. P. Pritzker, "The OARSI histopathology initiative - recommendations for histological assessments of osteoarthritis in the rabbit," Osteoarthritis and Cartilage, vol. 18, Supplement 3, pp. S53-S65, 2010.

[23] M. B. Goldring, K. Tsuchimochi, and K. Ijiri, "The control of chondrogenesis," Journal of Cellular Biochemistry, vol. 97, no. 1, pp. 33-44, 2006.

[24] L. Zhang, C. Liu, H. Wang et al., "Thrombopoietin knock-in augments platelet generation from human embryonic stem cells," Stem Cell Research \& Therapy, vol. 9, no. 1, article 194, 2018.

[25] H. Yu, G. Cauchois, J. F. Schmitt et al., "Is there a cause-andeffect relationship between physicochemical properties and cell behavior of alginate-based hydrogel obtained after sterilization?," Journal of the Mechanical Behavior of Biomedical Materials, vol. 68, pp. 134-143, 2017.

[26] H. Cao, Z. Yue, H. Gao et al., "In vivo real-time imaging of extracellular vesicles in liver regeneration via aggregationinduced emission luminogens," ACS Nano, vol. 13, no. 3, pp. 3522-3533, 2019.

[27] Q. Wu, L. Zhang, P. Su et al., "MSX2 mediates entry of human pluripotent stem cells into mesendoderm by simultaneously suppressing SOX2 and activating NODAL signaling," Cell Research, vol. 25, no. 12, pp. 1314-1332, 2015.

[28] W. Zhang, C. Liu, D. Wu et al., "Decitabine improves platelet recovery by down-regulating IL-8 level in MDS/AML patients with thrombocytopenia," Blood Cells, Molecules \& Diseases, vol. 76, pp. 66-71, 2019.

[29] S. Zhang, Y. Liu, X. Zhang et al., "Prostaglandin $E_{2}$ hydrogel improves cutaneous wound healing via M2 macrophages polarization," Theranostics, vol. 8, no. 19, pp. 5348-5361, 2018.

[30] Y. Wei, Y. Wu, R. Zhao et al., "MSC-derived sEVs enhance patency and inhibit calcification of synthetic vascular grafts by immunomodulation in a rat model of hyperlipidemia," Biomaterials, vol. 204, pp. 13-24, 2019.

[31] E. A. Makris, A. H. Gomoll, K. N. Malizos, J. C. Hu, and K. A. Athanasiou, "Repair and tissue engineering techniques for articular cartilage," Nature Reviews Rheumatology, vol. 11, no. 1, pp. 21-34, 2015.
[32] L. L. Lu, Y. J. Liu, S. G. Yang et al., "Isolation and characterization of human umbilical cord mesenchymal stem cells with hematopoiesis-supportive function and other potentials," Haematologica, vol. 91, no. 8, pp. 1017-1026, 2006.

[33] F. Barry and M. Murphy, "Mesenchymal stem cells in joint disease and repair," Nature Reviews Rheumatology, vol. 9, no. 10, pp. 584-594, 2013.

[34] Y. Zhang, Y. Li, W. Li et al., "Therapeutic effect of human umbilical cord mesenchymal stem cells at various passages on acute liver failure in rats," Stem Cells International, vol. 2018, Article ID 7159465, 11 pages, 2018.

[35] A. Tyndall, "Mesenchymal stem cell treatments in rheumatology: a glass half full?," Nature Reviews Rheumatology, vol. 10, no. 2, pp. 117-124, 2014.

[36] M. Loibl, K. Wuertz-Kozak, G. Vadala, S. Lang, J. Fairbank, and J. P. Urban, "Controversies in regenerative medicine: should intervertebral disc degeneration be treated with mesenchymal stem cells?," JOR Spine, vol. 2, no. 1, article e1043, 2019.

[37] P. Menasche, "Cell therapy trials for heart regeneration - lessons learned and future directions," Nature Reviews. Cardiology, vol. 15, no. 11, pp. 659-671, 2018.

[38] G. M. van Buul, M. Siebelt, M. J. Leijs et al., "Mesenchymal stem cells reduce pain but not degenerative changes in a mono-iodoacetate rat model of osteoarthritis," Journal of Orthopaedic Research, vol. 32, no. 9, pp. 1167-1174, 2014.

[39] A. T. Wang, Y. Feng, H. H. Jia, M. Zhao, and H. Yu, “Application of mesenchymal stem cell therapy for the treatment of osteoarthritis of the knee: a concise review," World Journal of Stem Cells, vol. 11, no. 4, pp. 222-235, 2019.

[40] Y. B. Park, M. Song, C. H. Lee, J. A. Kim, and C. W. Ha, "Cartilage repair by human umbilical cord blood-derived mesenchymal stem cells with different hydrogels in a rat model," Journal of Orthopaedic Research, vol. 33, no. 11, pp. 15801586, 2015.

[41] K. Brewer, B. Gundsambuu, P. Facal Marina, S. C. Barry, and A. Blencowe, "Thermoresponsive poly( $\varepsilon$-caprolactone)-poly(ethylene/propylene glycol) copolymers as injectable hydrogels for cell therapies," Polymers, vol. 12, no. 2, p. 367, 2020.

[42] S. Das, D. K. Sharma, S. Chakrabarty, A. Chowdhury, and S. Sen Gupta, "Bioactive polymersomes self-assembled from amphiphilic PPO-glycopolypeptides: synthesis, characterization, and dual-dye encapsulation," Langmuir, vol. 31, no. 11, pp. 3402-3412, 2015.

[43] E. Gu, W. Y. Chen, J. Gu, P. Burridge, and J. C. Wu, "Molecular imaging of stem cells: tracking survival, biodistribution, tumorigenicity, and immunogenicity," Theranostics, vol. 2, no. 4, pp. 335-345, 2012.

[44] M. Rodriguez-Porcel, "In vivo imaging and monitoring of transplanted stem cells: clinical applications," Current Cardiology Reports, vol. 12, no. 1, pp. 51-58, 2010.

[45] E. J. Oh, H. W. Lee, S. Kalimuthu et al., "In vivo migration of mesenchymal stem cells to burn injury sites and their therapeutic effects in a living mouse model," Journal of Controlled Release, vol. 279, pp. 79-88, 2018.

[46] Y. B. Park, C. W. Ha, J. A. Kim, S. Kim, and Y. G. Park, "Comparison of undifferentiated versus chondrogenic predifferentiated mesenchymal stem cells derived from human umbilical cord blood for cartilage repair in a rat model," The American Journal of Sports Medicine, vol. 47, no. 2, pp. 451461, 2019. 